Estuoos RBEEP

\title{
Estratégias de aprendizagem: reflexões sobre universitários
}

\author{
Afonso Galvão \\ Jacira Câmara \\ Michelle Jordão
}

\begin{abstract}
Resumo
Explora o tema estratégias de aprendizagem e revisa algumas pesquisas sobre a aprendizagem de estudantes universitários de diversos cursos de graduação. Especificamente, focaliza-se o estudo individual deliberado, a lei da potência de aprendizagem, estratégias individuais de estudo, a dimensão superficialidade-profundidade e as influências da avaliação no estudo individual. O estudo conclui que estudantes de graduação utilizam estratégias superficiais, qualitativa e quantitativamente ineficientes, que tendem a ser influenciadas pelo tipo de aula que vivenciam e pela dinâmica curricular que orienta a ação pedagógica nos cursos de graduação. Para superar isso é necessário que se estabeleça uma nova cultura de ensino e aprendizagem que leve em conta o modo como experts aprendem.
\end{abstract}

Palavras-chave: expertise; metacognição; autorregulação; avaliação. 


\section{Abstract \\ Learning strategies: reflections on undergraduate students}

This article explores the topic of learning strategies and review research on the learning of undergraduate students of several courses. Particularly, it is focused the individual learning practices, the ubiquitous law of practice, learning strategies, the surface-deep learning dimensions and the influence of learning assessment on individual practice. The study concludes that undergraduate students tend to use surface approaches which are inefficient in terms of both quality and quantity. These tend to be influenced by the teachers' practices as well as the curriculum dynamics that orients the pedagogic action of undergraduate courses. To overcome this, it is necessary to set up a new culture of teaching and learning which takes into account the ways in which experts learn.

Keywords: expertise; metacognition; self-regulation; learning assessment.

Para a maior parte das áreas acadêmicas (física, química, idiomas, crítica literária, pedagogia, psicologia, entre outras), o desenvolvimento da aprendizagem para a expertise começa quando da entrada do aprendiz em um curso de nível superior. Processos de aprendizagem, para serem eficientes, têm de levar em conta uma série de aspectos relacionados à estrutura curricular de um curso, às estratégias de aprendizagem de alunos, ao ambiente de aprendizagem e aos resultados de aprendizagem, expressos pela apropriação do discurso acadêmico por estudantes em modos aceitáveis de reflexão e prática de um tema, assunto, conteúdo ou área (Biggs, 1987). O estudo individual deliberado focalizado em uma área específica é tido por diversos autores (Galvão, 2003; Ericsson, 1996; Ericsson, Tesch-Römer, Krampe, 1993) como um dos mais importantes, senão o mais importante aspecto do desenvolvimento da expertise. Aqui, estratégias de aprendizagem constituem-se num detalhe particularmente importante, pois influenciam de modo direto a qualidade da expertise a ser desenvolvida. Envolvem, do ponto de vista do indivíduo, a interação entre aspectos metacognitivos e autorreguladores, que, por sua vez, são influenciados pela estrutura curricular das disciplinas ou matérias acadêmicas e do curso como um todo e pelas percepções do estudante sobre ambiente de ensino e de aprendizagem. Este artigo revisa teorias, conceitos e ideias em aprendizagem e os articula com pesquisas recentes que focalizam estratégias de aprendizagem de estudantes de graduação de vários cursos (Matemática, Psicologia, Letras-inglês e Pedagogia), 
buscando analisar a efetividade dessas estratégias e como elas se localizam na dimensão superficialidade-profundidade.

\section{Estudo deliberado}

O estudo individual deliberado constitui um termo usado por Ericsson, Tesch-Römer e Krampe (1993) para definir uma atividade de estudo em que o aprendiz não somente controla, como também toma as iniciativas do processo. A meta explícita desse tipo de estudo individual é a melhoria do desempenho. O estudo individual deliberado tem sido constantemente definido em relação à obtenção de alguma habilidade. Pear (1927), em um estudo clássico, argumenta que a habilidade está relacionada à quantidade e à qualidade do produto final, daquilo que emerge. À exceção de algumas atividades, como andar, correr e controlar movimentos do corpo próprio, que nascem com a pessoa, as habilidades - isto é, a capacidade para desempenhar bem uma tarefa -, em geral, são aprendidas. O estudo individual é a organização e apreensão de uma habilidade passo a passo. Trata-se do processo por meio do qual objetivos de aprendizagem são alcançados e transformados em ações rotineiras, muitas vezes, mas nem sempre, automatizadas.

O estudo individual para a aquisição de uma habilidade envolve a identificação de passos de ação que são componentes sequenciais ou alternados da habilidade, novos ou conhecidos do aprendiz, que se tornam uma habilidade total quando operados de forma compósita (Gagné, 1985). Alguns autores (e.g. Fitts, Posner, 1973; Gagné, 1985) identificam certas fases fundamentais na aquisição de uma habilidade, tais como: 1) compreensão dos comportamentos iniciais ou de entrada, que correspondem a um estágio cognitivo inicial; 2) organização de procedimentos de regras que determinam sequências, envolvendo a prática de partes de uma habilidade antes de tentá-la de um modo completo; e 3) o domínio final da tarefa.

Um dos aspectos mais importantes do estudo individual deliberado diz respeito à dimensão temporal, tema com importância histórica no contexto da pesquisa sobre aprendizagem, já que os primeiros trabalhos mais sistemáticos nesta área endereçaram este assunto. Podem ser incluídos aqui os estudos de Brian e Harter $(1897,1899)$ sobre a aprendizagem da linguagem telegráfica e outras pesquisas desenvolvidas no começo do século 20 (e.g. Starch, 1912; Pyle, 1913; 1914) os quais concluíram que a aprendizagem se beneficia mais da prática distribuída do que da prática concentrada. Além disso, intervalos prolongados de descanso seguidos de sessões curtas de estudo são mais eficientes para a aprendizagem. Em apoio a esta perspectiva, o argumento de Hull (1943) de que o incremento de reação de inibição é um fenômeno que trabalha contrariamente à potencialidade de reação da resposta presente ainda é atual - isto parece ser causado por períodos extensos de trabalho sem descanso. Adams (1987) considera que a reação inibidora é uma aversão que espontaneamente se 
dissipa como função do tempo entre respostas. No entanto, longos períodos de descanso após o estudo concentrado causam maior reminiscência devido à dissipação da reação inibidora.

O problema da relação idade/maturação/aprendizagem foi abordado em pesquisa também clássica de Wheeler e Perkins (1932). Este trabalho relaciona o fenômeno da aquisição de uma habilidade com o fenômeno da maturação e considera a questão do desenvolvimento de uma habilidade em relação ao tempo de maturação. Mencionam ainda Wheeler e Perkins que, em uma dada idade, o estudo deliberado traz melhoras na aprendizagem relacionadas ao tempo total de estudo individual desde que o estudo começou, ao invés de relacionadas a um período específico.

A dimensão "tempo de estudo" é particularmente relevante na pesquisa sobre aprendizagem para a expertise. Investigações longitudinais em uma variedade de áreas têm demonstrado de modo cada vez mais convincente que diferenças nas primeiras experiências de vida, preferências, hábitos, oportunidades e, principalmente, tempo de estudo individual deliberado qualitativa e quantitativamente eficiente são os principais determinantes da obtenção da capacidade performática expert, e não alguma possível habilidade diferenciadora de natureza genética. Por exemplo, para alguém se tornar jogador de xadrez capaz de disputar torneios internacionais são necessários pelo menos dez anos de preparação sistemática (Simon, Chase, 1973). Na composição musical, a preparação pode levar quase vinte anos (Hayes, 1981), enquanto que, na música instrumental, o estudo de Ericsson, Tesch-Römer e Krampe (1993) revelou que violinistas estudantes de conservatório superior atingem em torno de dez mil horas de estudo individual deliberado com 21 anos de idade. Em geral, pesquisas têm confirmado que, na maioria das áreas, são necessários pelo menos dez anos de estudo individual deliberado para que a expertise seja alcançada. Isso é particularmente verdade para a música instrumental (Sosniak, 1985; 1990), matemática (Gustin, 1985), tênis (Monsaas, 1985), ciência e literatura (Lehman, 1953) e atividade de ensino ou expertise de professores (Galvão, Perfeito [no prelo]).

Estes resultados, juntamente com outras investigações na área de estudos da expertise, ajudaram a reforçar a ideia de que o conhecimento expert é consequência de estudo individual deliberado de longo prazo em um domínio específico do conhecimento. Estas pesquisas também serviram para sustentar o desenvolvimento da "lei de potência da aprendizagem"; trata-se de uma das poucas leis da psicologia da aprendizagem, amplamente demonstrada, válida tanto para a performance motora quanto para a performance cognitiva. Esta lei explica o estudo deliberado como sendo a base do processamento automático - pode ser aplicada a qualquer situação de aprendizagem. De acordo com a lei ampla do estudo deliberado, da grafia de uma coordenada de eixos logarítmicos relacionando tempo por tentativa com número de tentativas resulta uma linha reta. Em outras palavras, o tempo que se leva para realizar uma performance diminui à medida que a prática se desenvolve (Newell, Rosenbloom, 1981). Esta linha reta relacionando acúmulo de prática a desempenho foi 
demonstrada em uma grande quantidade de tarefas motoras e cognitivas (Anderson, 2005). A Figura 1 mostra um gráfico representativo da lei da potência de aprendizagem, bem como sua expressão matemática.

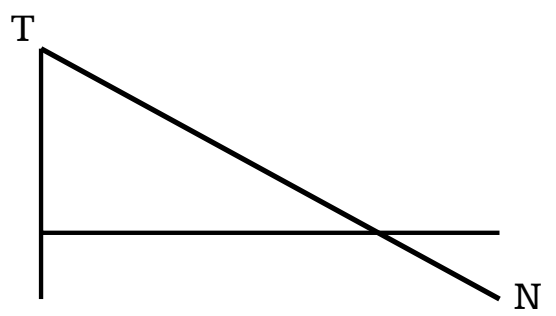

Figura 1 - Lei de potência da aprendizagem: tempo de performance diminui com a prática

Fonte: Adaptado de Newell e Rosenbloom (1981).

$\log (T)=\log (B)-\stackrel{\circ}{\log (N)}$

(B) é o tempo de performance quando da primeira tentativa.

(T) é o tempo, que é uma função de (N) que é o número de tentativas.

(N) é um modo de marcar o contínuo temporal (t) em intervalos temporais de performance. å é a razão de aprendizagem.

Apesar da importância do estudo deliberado para a aprendizagem, tal como demonstrada pela lei da potência de aprendizagem, infelizmente estudantes parecem não perceber isso. Pesquisas realizadas com estudantes de graduação de vários cursos (Matemática, Psicologia, Letras-inglês e Pedagogia) demonstram que a maioria dos acadêmicos tem dedicado pouco tempo para a aprendizagem fora da sala de aula. No geral, 39\% dos estudantes participantes das pesquisas dedicam de uma a duas horas de estudo diárias e $24,5 \%$ dedicam apenas uma hora do dia para o estudo individual deliberado. De acordo com Galvão (2001), há considerável defasagem entre o tempo de dedicação deliberada necessária à preparação de experts, evidenciado por pesquisas da área (Galvão, 2000; Ericsson, Tesch-Römer, Krampe, 1993), e o tempo de dedicação de estudantes universitários. Os resultados das pesquisas com universitários confirmam essa diferença e sugerem a necessidade de se trabalhar a dimensão temporal do estudo junto aos estudantes.

\section{Estudo superficial e profundo}

Um aspecto conceitual importante do estudo diz respeito à diferença entre procedimentos superficial e profundo de estudo individual. Esta distinção tem sido proposta por diversos autores (e.g. Mcune, Entwistle, 2000; Marton, Saljo, 1997) para caracterizar o modo como estudantes abordam tarefas acadêmicas. Enquanto abordagens profundas envolvem buscar significação, relacionar ideias, usar evidência e interessar-se por ideias, abordagens superficiais dizem respeito a falta de entendimento, falta de propósito de estudo e motivação relacionada apenas a medo de fracasso. Pesquisa de Marton e Saljo (1976) explorou as abordagens de 
aprendizagem de um grupo de estudantes em relação a uma tarefa que envolvia a leitura de um texto acadêmico tendo em vista a resposta a um determinado conjunto de perguntas sobre ele.

Os resultados indicaram que, para ler, os alunos se relacionavam com essa tarefa basicamente de duas formas. A primeira focalizando a compreensão do texto como um todo e a segunda focalizando a memorização e a reprodução de conteúdos conceituais do texto. Essas duas abordagens em relação à aprendizagem influenciaram os conceitos de "abordagem profunda" e "abordagem superficial" (Biggs, 2003).

$\mathrm{Na}$ abordagem profunda, o estudante focaliza a sua atenção no esforço de análise e compreensão de conceitos e princípios do objeto ou tema de aprendizagem; para tanto, tende a adotar estratégias que o ajudem a satisfazer a sua curiosidade na procura do significado inerente à tarefa, de modo a personalizá-la e a torná-la mais coerente com a sua experiência, integrando-a em conhecimentos adquiridos antes ou teorizando acerca da possibilidade dessa integração, levantando hipóteses, regulando os aspectos contraditórios nelas implicados (Bessa, Tavares, 2000).

Na abordagem superficial, ao contrário, o estudante adota uma atitude passiva de aceitação das informações fornecidas pelos professores e materiais didáticos e/ou memorização e reprodução de temas abordados, desarticulado de compreensão efetiva. Quando estudantes adotam essa abordagem, a estratégia geral consiste em focar o essencial dos conteúdos ou matéria de estudo, normalmente informação factual e concreta, havendo a preocupação de representá-la simbolicamente também dessa forma, com vista à sua reprodução o mais fiel e exato possível (Bessa, Tavares, 2000).

A memorização, estratégia superficial, caracteriza-se pela utilização de repetição enquanto procedimento para armazenamento da informação. Segundo Duarte (2002), os elementos literalmente memorizados são mais tarde "regurgitados" nas avaliações exigidas pelos processos acadêmicos. A utilização de estratégia superficial conduz para uma retenção restrita e temporária da informação. Cabe considerar, no entanto, que reproduzir e memorizar desempenham no discurso do ato de aprender um papel importante, porém insuficiente, já que compreender vai além da transformação do conhecimento apresentado: trata-se da capacidade relacional com o que já se sabe, promovendo a compreensão e aplicação para a vida.

Entwistle e Smith (2002) distinguem entre entendimento alvo e entendimento pessoal. Entendimento alvo diz respeito ao tipo de entendimento que professores, examinadores e elaboradores de currículo têm em mente quando organizam um currículo para ser estudado, enquanto que entendimento pessoal diz respeito àquilo que na verdade os estudantes desenvolvem ou alcançam. Estudantes universitários trazem para qualquer curso ou disciplina em que se matriculam compreensões e experiências desenvolvidas em suas vivências educacionais e informais anteriores que influenciam o modo como fazem sentido do assunto presente e seu estudo individual. Além disso, estudantes ingressam na universidade com hábitos de estudo já estabelecidos, alguns dos quais 
inapropriados para a educação superior. Nesta perspectiva, uma dada situação de aprendizagem pode ser interpretada pelo aluno a partir da sua experiência prévia, onde a regulação externa tende a ser mais forte, envolvendo orientações sobre como e o que trabalhar. A aprendizagem para a expertise, que acontece em nível superior, depende de procedimentos marcadamente autorregulados.

Dessa forma, as concepções dos estudantes sobre aprendizagem influenciam significativamente a forma como enfrentam as tarefas escolares. Segundo Van Rossum e Schenck (1984), alunos "superficiais" apresentam geralmente concepções de aprendizagem quantitativas (como aumento de conhecimento, memorização, reprodução para aplicar), enquanto os alunos que optam pela abordagem profunda apresentam concepções qualitativas (aprender é compreender, ver as coisas de forma diferente, mudar como pessoa). Para alterar a abordagem à aprendizagem é necessário, entre outros aspectos, promover concepções de aprendizagem qualitativamente mais profundas por meio de contextos de aprendizagem estimulantes (Ramsden, Beswick, Bowden, 1986).

Na mesma direção, afirma Ramsden (1992) que as diferentes tarefas e contextos impõem aos alunos a utilização de estratégias "ajustadas" de estudos e aprendizagens. Ou seja, os estudantes tendem a adaptar-se, o melhor possível, às exigências e oportunidades que lhes são apresentadas. Dessa forma, o autor apresenta três domínios contextuais que influenciam o desenvolvimento de estratégias de estudo e aprendizagem e contexto acadêmico: o método usado pelos professores para facilitar a aprendizagem dos alunos - o ensino; o método utilizado pelo professor para avaliar aquilo que é aprendido - a avaliação; e o conteúdo e a estrutura daquilo que formata a aprendizagem em cada disciplina - os programas.

Segundo Zabalza (2002), o problema é que estudantes comumente não possuem informações sobre técnicas de estudo, além de carência de estratégias para lidar com as atividades educativas propostas. Porém, não cabe aos estudantes resolverem essa situação na totalidade, pois é difícil que estes aprendam a aprender se essa questão não constitui objetivo formativo e não há orientação por parte do professor.

Nessa perspectiva, o professor é considerado um ponto essencial no contexto de aprendizagem. Cabe a ele ou a ela coordenar o processo educativo, incluindo estratégias de ensino e de avaliação fundamentadas na metacognição e na autorregulação. Assim, uma das tarefas educativas do professor é a construção de ambientes de aprendizagem profundos (ativos e motivadores), onde é dada ao estudante a oportunidade de aprender a pensar, criticar, raciocinar e questionar certezas, incluindo a metacerteza das verdades garantidas pelo acesso a métodos científicos. Como argumenta Freire (1997), as dúvidas que movimentam a evolução da ciência devem constituir parte fundamental do processo educativo. Assim, é preciso substituir as certezas e saberes preestabelecidos na lógica simplista que constitui a pedagogia de hoje por outra, complexa, que trabalhe com conceitos transversais, abertos para a surpresa e o imprevisto (Assmann, 1996). Para isso, é necessário que o professor mude o foco de 
si para o estudante. Como argumenta Entwistle (2000), professores que conduzem aulas tendo a si próprios como centro e o conteúdo como foco tendem a influenciar seus alunos a adotarem estratégias superficiais de aprendizagem. Por outro lado, quando o foco do ensino está no estudante, a aprendizagem gera transformação e estimula a utilização de estratégias profundas.

\section{Estratégias de aprendizagem utilizadas por estudantes de graduação}

Estratégias são consideradas variáveis importantes na educação e influenciam na qualidade da aprendizagem e, portanto, têm sido objeto de variadas pesquisas (Pozo, 2002; Weinstein, Mayer, 1986; Monereo et al., 1995; Flavell, 1999).

Para Monereo et al., (1995, p. 25), o termo estratégia de aprendizagem está relacionado a processos de tomada de decisão pelos quais o aprendiz escolhe e recupera, de maneira organizada, "os conhecimentos de que necessita para completar um determinado objetivo, dependendo das características da situação educativa na qual se produz a ação". As estratégias não podem ser confundidas com as técnicas de aprendizagem, pois são procedimentos que se aplicam de modo controlado, dentro de um plano projetado deliberadamente com o objetivo de se obter uma meta (Pozo, 2002).

Weinstein e Mayer (1986) relacionam estratégias de aprendizagem a ensaio, elaboração, organização e monitoramento. As estratégias de ensaio visam à repetição ativa da fala e/ou da escrita do material a ser aprendido; as estratégias de organização implicam a estruturação do material a ser aprendido, subdividindo-o em partes reconhecidas nas relações subordinadas ou superordenadas. A elaboração envolve a reconstrução significativa de um material de aprendizagem. O objeto estímulo da aprendizagem torna-se integrado ao esquema conceitual do aprendiz possibilitando novos usos em diferentes contextos. O monitoramento é o feedback, a princípio externo, que, torna-se gradualmente em auto-feedback à medida que a expertise se desenvolve.

As estratégias de aprendizagem visam, pois, auxiliar os processos de aprendizagens dos estudantes, tendo como objetivo a melhoria do desempenho escolar. Nesse sentido, a palavra "estratégia" diz respeito a planejamento e procedimentos para alcançar a aprendizagem. Flavell (1999) diferencia os tipos de estratégias em duas dimensões: cognitiva, destinada simplesmente a levar o estudante a um objeto cognitivo, e metacognitiva, que se propõem avaliar a eficácia da primeira, ou seja, é operacionalizada como monitoração da compreensão, que requer o estabelecimento de objetivos de aprendizagem, a avaliação do grau de compreensão que se deseja alcançar e, se necessário, a modificação das estratégias utilizadas.

Resultados de pesquisas sobre estratégias de aprendizagem de estudantes de graduação em Matemática (Pereira, 2007), Letras-inglês 
(Bastos, 2007), Pedagogia (Demoliner, 2008) e Psicologia (Lima, 2007), que investigaram como o aprendiz aborda seus estudos, técnicas que utiliza e hábitos adotados, apontam que as estratégias desenvolvidas, por vezes convergentes nos quatro cursos analisados, nem sempre são as mais adequadas para o processo de aprendizagem.

Estudar fazendo anotações é uma estratégia tida como bastante eficiente por diferentes autores em aprendizagem (Craik, Lockhart, 1975; Galvão, 1999; Eysenck, Keane, 1994). Esta estratégia é condizente com uma teoria influente em pesquisas cognitivas da memória - a dos níveis de processamento (Craik, Lockhart, 1975). De acordo com esta teoria, o material armazenado na memória de longa duração é determinado por processos perceptuais e atencionais que ocorrem durante a fase de aprendizagem. Tais processos ocorrem em diferentes níveis e podem variar desde análises superficiais de um estímulo até análises semânticas profundas (Eysenck, Keane, 1994). Em outras palavras, a memória de um evento é fortemente influenciada pela profundidade de processamento de um estímulo - quanto mais profundo o nível de processamento, mais forte, elaborado e longo o traço de memória. Isto é complementado pela ideia de que a elaboração do processamento, no que concerne a quantidade (Craik, Tulving, 1975) e tipo (Bransford et al., 1979), tende a ser fortemente influenciada por pistas estruturadas em formas múltiplas de processamento (com elaboração monitorada). Pesquisa de Galvão (1999) mostrou as implicações dessa teoria para a aprendizagem ao demonstrar que a leitura de textos fazendo anotações reforça significantemente o traço de memória do conteúdo textual. Uma possível explicação para isso é que esta estratégia de aprendizagem envolve processamento ortográfico e fonológico, o que remete a domínios auditivos e visuais. Estudar fazendo anotações é uma estratégia utilizada por estudantes dos quatro cursos investigados (Psicologia, 67\%; Pedagogia, 61\%; Letras-inglês, 60\%; Matemática, 52\%), embora haja alguma variação no percentual de uso das estratégias entre os cursos. Uma possível explicação para o menor percentual em Matemática talvez derive do fato de que nessa área do conhecimento se utiliza menos textos.

A leitura de outras fontes não relacionadas ao curso também é uma estratégia de aprendizagem bastante utilizada pelos estudantes de graduação. A pesquisa e a leitura normalmente ocorrem em fontes diversas e auxiliam uma estratégia de estudo de abordagem profunda, por meio da busca do conhecimento de forma contextualizada e multidisciplinar. Esta estratégia é evidenciada nas respostas dos estudantes das graduações pesquisadas, quando afirmaram que sempre ou com frequência realizam seus estudos desta forma (Psicologia, 64\%; Matemática, 58,9\%; Pedagogia, 58,6\%; Letras-inglês, 57\%). Entwistle (2000) sugere que a intenção de extrair significado leva a processos de aprendizagens ativos que envolvem, por um lado, relacionar ideias e procurar padrões e princípios e, por outro, utilizar evidências e examinar a lógica argumentativa. Dessa forma, os aprendizes que adotam esta estratégia estão predominantemente motivados pelo interesse ao objeto de estudo e/ou 
reconhecem a sua relevância e procuram relacionar a matéria com seu conhecimento prévio e experiências pessoais.

A estratégia de aprendizagem baseada na resolução de problemas é considerada essencial para o estudo profundo, tendo em vista que desenvolve uma habilidade reflexiva e crítica sobre o próprio raciocinar. Oportuniza o pensar sobre as cognições, o comportamento e a própria aprendizagem, inclui a autorregulação da aprendizagem, ou seja, envolve, da parte do aprendiz, tomar consciência sobre objetivos de estudo para organizar e dirigir o próprio processo de aprendizagem (Weidenbach, 1996). Para Zimmerman e Martinez-Pons (1986), estudantes autorregulados são metacognitivos, motivados e participantes ativos nos seus processos de aprendizagem; tornam-se agentes de sua própria aprendizagem, pois iniciam e dirigem seus próprios esforços para aquisição de conhecimentos e desenvolvimento de habilidades. No entanto, segundo os dados das pesquisas, o hábito de estudar resolvendo exercícios é sempre ou frequentemente utilizado somente no curso de Matemática $(82,1 \%)$, em cuja formação predomina as disciplinas das ciências exatas. Nos demais cursos analisados, a prática de estudar resolvendo problemas apresenta percentuais relativamente baixos, como se pode verificar, por exemplo, no curso de Psicologia, onde 37,4\% dos entrevistados afirmaram que nunca ou raramente estudam por meio dessa estratégia.

Outras estratégias de estudo que merecem consideração são aquelas que denotam uma aprendizagem superficial dos conteúdos. Decorar conteúdos, por exemplo, é um tipo de estudo individual utilizado frequentemente por 38,8\% dos participantes da área de Matemática e sempre por parte considerável dos estudantes de Letras (33,3\%), Pedagogia (27\%) e Psicologia (27,1\%). Isso sugere que a prática da famosa e questionável "decoreba" ainda está presente em nosso cotidiano acadêmico. Fazer leitura prévia dos conteúdos também não é um hábito de estudo frequente dimensionado pelos estudantes de graduação (Letras-inglês, 48,7\%; Psicologia, 46\%; Pedagogia, 38,5\%; Matemática, 36,4\%), assim como rever os conteúdos, essencial para abordagem profunda (Letras, 31,7\%; Pedagogia, 35,6\%; Psicologia, 34,5\%; Matemática, 34,9\%). Observa-se ainda que a revisão de conteúdos, além de não ser uma prática constante no hábito de estudos de cursos na área de humanas, faz-se, quando é desenvolvida, de maneira segmentada (Letras, 43,5\%; Pedagogia, 36,2\%; Psicologia, 43\%).

Estratégias que induzem os estudantes à memorização, ao acúmulo de informação desordenadas e desarticuladas estão diretamente associadas à aprendizagem deficiente e superficial (Perkins, 1991). De acordo com Stiernborg, Guy e Tinker (1997), os alunos que adotam a estratégia superficial são motivados predominantemente pela necessidade de atender às expectativas de um determinado curso ou pelo medo do fracasso escolar. Neste caso, a intenção é meramente a de realizar tarefas através da utilização de fragmentos de informações ou ideias sem relações entre si, levando a processos de aprendizagem ainda mais restritos, tais como a memorização e a aprendizagem automática (Emilia, Mulholland, 1991). 
No entanto, cabe ressaltar que, apesar de a reprodução e a memorização desempenharem, no decurso do ato de aprender, papéis necessários, são insuficientes para se obter a capacidade de relacionar o que se sabe com o que será aprendido, operação fundamental na constituição da aprendizagem significativa.

Pelos dados apresentados nessas pesquisas, constatou-se ainda que os estudantes não tenham por hábito fazer qualquer tipo de preparação física ou mental para realizarem o estudo individual deliberado, fato que pode ser verificado pelos respondentes que nunca fazem uso desta proposição: Pedagogia, 47,2\%; Matemática, 42,6\%; Letras-inglês, 33,9\%. Para Jaime (2001), a preparação física e/ou mental é um dos fatores que beneficiam o processo de aprendizagem, uma vez que todas as estratégias de aprendizagem têm como base a evocação da resposta de relaxamento rápido. Assim sendo, os estudantes, durante a aprendizagem, estão em alerta relaxado ou vigília relaxado.

\section{Influências da avaliação no estudo individual deliberado}

As práticas de avaliação dos professores, segundo algumas pesquisas (Marton, Saljo, 1997; Biggs, 2003), também influenciam sobremaneira as abordagens de aprendizagem que os estudantes desenvolvem na graduação. Práticas avaliativas devem estar relacionadas a aspectos formativos, fundamentadas na autoaprendizagem e, portanto, na capacidade de assumir um posicionamento crítico sobre o próprio raciocinar, a fim de se tomar consciência sobre os seus objetivos do estudo para organizar e dirigir o próprio processo de aprendizagem. Trata-se da metacognição que envolve a tomada de consciência, por parte do aprendiz, sobre seus objetivos de estudo para organizar e dirigir o próprio processo de aprendizagem, bem como a autorregulação da aprendizagem (Weidenbach, 1996). Assim, aquisição de conhecimento implica aprendizes serem capazes de ir além do conhecimento factual para chegar à capacidade de pensar sobre os próprios pensamentos.

É importante então diferenciar dois conceitos que por vezes são considerados como sinônimos: a prática de exames e a prática de avaliação. O exame é um processo classificatório e, por conseguinte, seletivo e excludente, por não se preocupar em avaliar, mas tão somente em selecionar, classificar. Segundo Luckesi (2005), o exame, diferentemente da avaliação, que busca diagnosticar, subsidiar a tomada de decisões para a reorientação do processo pedagógico, possui uma visão estática do sujeito aprendiz. O que interessa é o "aqui e o agora" e não o que ele poderá vir a saber no futuro. Para o autor, a visão do exame está intrinsecamente associada à pedagogia tradicional, pois é fragmentada e pontual, por isso não sustenta a real prática avaliativa, que é processual, gradual, dinâmica, dialógica e inclusiva.

Para Moraes (2008), avaliar significa subsidiar e colaborar tanto para a melhoria qualitativa da aprendizagem quanto para uma melhor 
compreensão das dificuldades e dos problemas, bem como dos elementos facilitadores de tais processos. Assim, sua função primordial estaria em estabelecer uma parceria de cumplicidade e confiança entre educador e educando, bem como o desenvolvimento constante do aprender a aprender.

Dessa forma, o ato de avaliar a aprendizagem implica trabalhar de forma integrada e integradora, relacional, sujeita às emergências e não-linear, por meio de um currículo aberto, dinâmico e flexível. A avaliação, nesse contexto, deve estar a serviço da dimensão formadora e dinâmica, não apenas somativa. Ou seja, a avaliação daí decorrente não se isola em instrumento de verificabilidade, mas em experiência - tempo-espacial - de manifestação das potencialidades do ser humano e sua capacidade de crescer, desenvolver-se, aprimorar-se. Apesar dessas considerações em relação ao processo avaliativo, percebe-se que estudantes de cursos de graduação somente às vezes desenvolvem trabalhos exigidos pelos docentes que os ajudam a fazer conexões entre conhecimento adquirido com as experiências anteriores (Matemática, 46,5\%; Pedagogia, 48\%; Psicologia, 52,3\%; Letras-inglês, 43,6\%), a apreender algo relacionado entre si (Matemática, 49,6\%; Pedagogia, 44,3\%; Psicologia, 58\%; Letrasinglês, 45,3\%) e a estudar sabendo o para quê, ou seja, percebendo um objetivo claro de seus estudos (Matemática, 36,4\%; Pedagogia, 37,9\%; Psicologia, 50,5\%; Letras-inglês, 40\%).

Verifica-se que as práticas pedagógicas desenvolvidas nos cursos de graduação apresentados colocam a avaliação como encorajadora da memorização mecânica e do relembrar, sem relevância social e pessoal, tendendo a estimular estratégias de estudo mais superficiais. No entanto, fazem-se necessárias práticas avaliativas relacionadas à compreensão e ao potencial de uma aprendizagem de maior qualidade por meio de ações que visem à construção progressiva de objetivos pessoais e de um projeto de aprendizagem/vida embasados no incentivo da autorreflexão e da metacognição que podem influenciar a elaboração de concepções de aprendizagem mais complexas, e, consequentemente, de abordagens profundas ao aprender. Tal perspectiva visa, também, apresentar procedimentos e processos que permitam aos estudantes aprender a aprender, ponto crucial para a qualidade da aprendizagem, à medida que concebe o aprender de forma mais profunda e incentiva a regulação da própria aprendizagem.

\section{Conclusões}

As evidências das pesquisas citadas ao longo do texto sugerem uma cadeia de aspectos que lançam sérias dúvidas sobre a capacidade dos cursos de graduação de se tornarem realmente a etapa inicial da aprendizagem de experts. O estudo individual dos participantes da pesquisa, em geral, além de ser quantitativamente irrisório, tende a ser também superficial. Embora a responsabilidade e a disponibilidade dos estudantes 
para a aprendizagem não possa ser desconsiderada, grande parte das responsabilidades pelo uso de estratégias de aprendizagem que levam a resultados pífios é dos professores e da própria estrutura curricular dos cursos de graduação, que parecem não suscitar o raciocínio crítico e a reflexão sistemática. Aprendizagem, para ser eficiente, precisa ser reconstrutiva e ressignificativa; isso exige focalizar o processo de ensino no estudante e não no professor. Trata-se de incluir na prática pedagógica a ideia de que aprender é resolver problemas e ensinar, oferecer feedback. Aqui, práticas avaliativas devem ter como foco principal a análise de desempenho, permitindo a correção de aspectos indesejados. A boa prática avaliativa influencia o desenvolvimento do autofeedback, contribuindo sobremaneira para a consolidação da autonomia intelectual, aspecto fundamental do desempenho expert. Processos avaliativos, se bem orientados, levarão em conta o estabelecimento de diagnósticos específicos e gerais sobre a aprendizagem alcançada de acordo com as metas de aprendizagem estabelecidas. Uma avaliação desse tipo é estruturada, relacional, emergente, flexível, dinâmica, discursiva, capaz de suscitar não só aprendizagem significante, como também metacognição e autorregulação, aspectos fundamentais da aprendizagem autônoma.

Pessoas que se tornam experts têm acesso a experiências de aprendizagem desse tipo. Como sugerem muitas pesquisas (Sosniak, 1990; Ericsson, Tesch-Römer, Krampe, 1993; Galvão, 2003), experts, quando estudantes, tiveram acesso a sistemas de aprendizagem que privilegiavam feedbacks qualitativamente eficientes e constantes, cujas regras acabavam sendo internalizadas para se tornarem progressivamente em autofeedbacks eficientes. Isso ocorre evidentemente em contextos de aprendizagem que levavam em conta características individuais de aprendizes. Um problema sério aqui é que os custos de um sistema de aprendizagem desse tipo são financeiramente caros. Frequentemente envolve aulas em turmas pequenas ou mesmo do tipo um para um, como no caso da formação de mestrado ou doutorado. Há um problema de difícil solução, no entanto esta é uma aprendizagem talvez cara demais para a educação de massas. A construção de soluções capazes de gerar caminhos que se aproximem da aprendizagem expert é um dos grandes desafios da dinâmica curricular brasileira, na qual a avaliação deve ser considerada como um subsídio para o replanejamento do processo de ensino e aprendizagem, uma avaliação que engloba não apenas medida, mas também valor e qualidade - avaliação como meio e não como fim em si mesma. Aqui, é fundamental o respaldo das políticas públicas dando sustentação às ações desenvolvidas no processo educativo.

\section{Referências bibliográficas}

ADAMS, J. Historical review and appraisal of research on the learning, retention and transfer of human motor skills. Psychological Bulletin, v. 101, n. 1, p. 41-74, 1987. 
ANDERSON, J. Aprendizagem e memória: uma abordagem integrada.

Rio de Janeiro: LTC, 2005.

ASSMANN, H. Metáforas novas para reencantar a educação:

epistemologia e didática. Piracicaba: Ed. Unimep, 1996.

BASTOS, V. da F. G. Estratégias de estudo deliberado e percepções sobre o contexto de ensino na perspectiva de estudantes do curso de letras-inglês. 2007. Dissertação (Mestrado em Educação) Universidade Católica de Brasília, 2007.

BESSA, J.; TAVARES, J. Abordagens e estratégias de regulação do estudo em alunos do $1^{\circ}$ ano das licenciaturas de ciências e engenharias da Universidade de Aveiro. Psicologia, v. 14, n. 2, p. 173-188, 2000.

BIGGS, J. B. Student approaches to learning and studying. Hawthorn: Australian Council for Educational Research, 1987.

. Teaching for quality learning at university. Buckingham:

Open University Press, 2003.

BRANSFORD, J. D. et al. Some general constraints on learning and memory research. In: CERMAK, L. S.; CRAIK, F. I. M. (Eds.). Levels of processing inhuman memory. Hillsdale, NJ: Erlbaum, 1979. p. 331-354.

BRIAN, W. L.; HARTER, N. Studies on the telegraphic language: the acquisition of a hierarchy of habits. Psychological Review, v. 6, p. 345-375, 1899.

. Studies in the physiology and psychology of the telegraphic language. Psychological Review, v. 4, p. 27-53, 1897.

CRAIK, F.; LOCKART, R. Levels of processing: a framework for memory research. Journal of Verbal Learning and Verbal Behavior, v. 11, p. 671-684, 1975.

CRAICK, F.; TULVING, E. Depth of processing and the retention of words in episodic memory. Journal of Experimental Psychology: General, n. 104, p. 268-294, 1975.

DEMOLINER, V. L. Estratégias de Estudo e Percepções sobre o Ensino em Contexto Universitário dos Aprendizes de Pedagogia. 2008. Dissertação (Mestrado em Educação) - Universidade Católica de Brasília, 2008.

DUARTE, A. Aprendizagem, ensino e aconselhamento educacional: uma perspectiva cognitivo-motivacional. Porto, Portugal: Porto, 2002. 
EMILIA, O.; MULHOLLAND, H. Approaches to learning of students in an Indonesian medical school. Medical Education, v. 25, p. 462-470, 1991.

ENTWISTLE, N. Enhancing teaching and learning project. Edinburgh, 2000 .

ENTWISTLE, N; SMITH, C. Personal understanding and target understanding: mapping influences on the outcomes of learning. British Journal of Educational Psychology, v. 66, p. 21-38, 2002.

ERICSSON, K. A. The acquisition of expert performance: an introduction to some of the issues. In: ERICSSON, K. A. (Ed.). The road to excellence: the acquisition of expert performance in the arts and sciences, sports and games. Mahwah, NJ: Lawrence Erlbaum Associates, 1996. p. 1-50.

ERICSSON, K. A.; TESCH-RÖMER, C.; KRAMPE, R. The role of deliberate practice in the acquisition of expert performance. Psychological Review, v. 100, n. 3, p. 363-406, jul. 1993.

EYSENCK, M. W.; KEANE, M. T. Psicologia cognitiva: um manual introdutório. Porto Alegre: Artes Médicas, 1994.

FITTS, P.; POSNER, M. Human performance. London: Prentice Hall, 1973.

FLAVELL, J. H. Cognitive development: children's knowledge about the mind. Annual Review of Psychology, v. 50, p. 21-45, 1999.

FREIRE, Paulo. Pedagogia da autonomia: saberes necessários à prática educativa. São Paulo: Paz e Terra, 1997.

GAGNÉ, R. The conditions of learning. New York: Holt, Rinehart and Winston, 1985.

GALVÃO, A. Practice in orchestral life: an exploratory study on string players' learning processes. Ph.D. Thesis. The University of Reading, UK, 2000.

Learning processes of expert players. In: INTERNATIONAL CONFERENCE ON MUSIC PERCEPTION AN COGNITION, 6., 2001, Keele, UK. Proceedings of the Sixth... Keele, UK, 2001.

Pesquisa sobre expertise: perspectivas e limitações. Temas de Psicologia, v. 9, n. 3, p. 223-237, 2003.

GALVÃO, A.; PERFEITO, C. Pesquisa sobre expertise e expertise do professor: implicações. Revista Lusófona de Educação, 2012. [no prelo]. 
GALVÃO, Izabel. Henri Wallon: uma concepção dialética do desenvolvimento infantil. Petrópolis: Vozes, 1999.

GUSTIN, W. The development of exceptional research mathematicians. In: BLOOM, B. (Ed.). Developing talent in young people. New York: Balantine Books, 1985.

HAYES, J. The complete problem-solver. Philadelphia: Franklin Institute Press, 1981.

HULL, C. Principles of behavior. New York: Appleton Century, 1943.

JAIME, F. A revolução da aprendizagem: um novo sistema de aprendizagem para o desenvolvimento do cérebro e a expansão da mente. Goiânia: Kelps, 2001.

LEHMAN, H. Age and achievement. Princeton: Princeton University Press, 1953.

LIMA, W. R. Estratégias de estudo deliberado e percepções sobre o ensino da graduação em psicologia. 2007. Dissertação (Mestrado em Psicologia) - Universidade Católica de Brasília, 2007.

LUCKESI, Cipriano. Avaliação da aprendizagem escolar. São Paulo: Cortez, 2005.

MARTON, F; SALJO, R. On qualitative differences in learning, outcomes and processes. British Journal of Educational Psychology, v. 46, p. 4-11, 1976.

. Approaches to learning. In: MARTON, F.; HOUNSELL, D. J.; ENTWISTLE, N. J. (Eds.). The experience of learning. 2. ed. Edinburgh: Scottish Academic Press, 1997. p. 39-58.

MCUNE, V; ENTWISTLE, N. The deep approach to learning: analytic abstraction and idiosyncratic development. Paper presented at Innovations in Higher Education Conference, Helsink, Finland, 2000.

MONEREO, C. et al. Estratégias de enseñanza y aprendizaje: formación del profesorado y aplicación en la escuela. Barcelona: Grão Editorial, 1995.

MONSAAS, J. Learning to be a word-class tennis player. In: BLOOM, B.

(Ed.). Developing talent in young people. New York: Balantine Books, 1985.

MORAES, Maria Cândida. Pensamento eco-sistêmico: educação,

aprendizagem e cidadania no século XXI. 2. ed. Petrópolis: Vozes, 2008. 
NEWELL, A.; ROSENBLOOM, P. Mechanisms of skill acquisition and the law of practice. In: ANDERSON, J. (Ed.). Cognitive skills and their acquisition. Hillsdale, NJ: Erlbaum, 1981.

PEAR, T. H. Skill. Journal of Personnel Research, v. 5, p. 478-489, 1927.

PEREIRA, M. A. Estratégias de estudo deliberado e percepções sobre o ensino de matemática em contexto universitário. 2007. Dissertação (Mestrado em Educação) - Universidade Católica de Brasília, 2007.

PERKINS, D. What constructivism demands of the learner. Educational Researcher, v. 18, p. 16-25, 1991.

POSNER, M. I.; RAICHLE, M. E. Imagens da mente. Porto, Portugal: Porto, 2001.

POZO, J. I. Aprendizes e mestres: a nova cultura da aprendizagem. Porto Alegre: Artes Médicas, 2002.

PYLE, W. Economical learning. Journal of Educational Psychology, v. 4, p. 148-158, 1913.

Concentrated versus distributed practice. Journal of Educational Psychology, v. 5, p. 247-251, 1914.

RAMSDEN, P. Learning to teach in higher education. London: Kogan Page, 1992.

RAMSDEN, P.; BESWICK, D.; BOWDEN, J. Effects of learning skills intervention on first year university student's learning. Human Learning, v. 5, p. 151-164, 1986.

ROSARIO, P. Motivação e aprendizagem: uma rota de leitura. In: TAVEIRA M.C., (Coord.). Temas de Psicologia Escolar: contributos de um projecto científico-pedagógico. Coimbra: Quarteto, 2005. p. 23-60.

SIMON, H.; CHASE, W. Skills in chess. American Scientist, v. 61, p. 394-403, 1973.

SOSNIAK, L. Learning to be a concert pianist. In: BLOOM, B. S. (Ed.). Developing talent in young people. New York: Ballantine Books, 1985.

. The tortoise, the hare, and the development of talent. In: HOWE, M. (Ed.). Encouraging the development of exceptional skills and talents. Leicester: British Psychological Society, 1990.

STARCH, D. Periods of working in learning. Educational Psychology, v. 3, p. 209-213, 1912. 
STIERNBORG, M.; GUY, J.; TINKER, R. Nursing students approaches to studying. Nurse Education Today, v. 17, p. 121-127, 1997.

VAN ROSSUM, E. J.; SCHENCK. S. M. The relationship between learning conception, study strategy and learning outcome. British Journal of Educational Psychology, v. 54, n. 1, p. 73-85, 1984.

WEIDENBACH, V. The influence of self-regulation on instrumental music practice. Ph.D. thesis. University of Western Sydney, Nepean, 1996.

WEINSTEIN, C. E.; MAYER, R. F. The teaching of learning strategies. In: WITTROCK, M. C. (Ed.) Handbook of research on teaching. New York: Academic Press, 1986. p. 315-327

WHEELER, R.; PERKINS, F. Principles of mental development. New York: Crowell, 1932.

ZABALZA, Miguel A. O ensino universitário: seu cenário e seus protagonistas. Porto Alegre: Artmed, 2004.

. Os professores. Revista Pátio, v. 6, n. 22, p. 15-20, jul./ago. 2002.

ZIMMERMAN, B.; MARTINEZ-PONS, M. Development of a structured interview for assessing students use of self regulated learning strategies. American Educational Research Journal, v. 23, n. 4, p. 614-628, 1986.

Afonso Galvão, doutor em Educação pela Universidade de ReadingInglaterra, é coordenador do Programa de Pós-Graduação em Educação da Universidade Católica de Brasília (UCB).

acetaga@gmail.com

Jacira Câmara, doutora em Educação pelo Peabody College for Teachers at Vanderbilt - USA, é professora do Programa de Educação da Universidade Católica de Brasília (UCB).

jacira@ucb.br

Michelle Jordão, doutoranda em Educação pela Universidade Católica de Brasília (UCB).

michellem@ucb.br

Recebido em 28 de julho de 2011.

Aprovado em 18 de abril de 2012. 